\section{Interner Datenschutzbeauftragter: TÜV NORD und BITKOM kooperieren bei Ausbildung}

Der Bundesverband Informationswirtschaft, Telekommunikation und neue Medien (BITKOM) erhält beim Thema Ausbildung Unterstützung von der TÜV NORD Akademie und TÜV Informationstechnik (TÜViT). Die beiden Tochterunternehmen von TÜV NORD haben mit der Bitkom Servicegesellschaft einen Kooperationsvertrag geschlossen, der die Kompetenzen für die Ausbildung zum internen Datenschutzbeauftragten bündelt.

Der Datenschutzbeauftragte erfüllt im Multimedia-Zeitalter eine wichtige Funktion. Jedes Unternehmen, gleich welcher Größe oder Branche, das personenbezogene Daten automatisiert verarbeitet, unterliegt dem Bundesdatenschutzgesetz und hat die damit verbundenen Pflichten zu erfüllen. Eine wesentliche Aufgabe des internen Datenschutzbeauftragten ist die Kontrolle und Überwachung der ordnungsgemäßen Anwendung von Datenverarbeitungsprogrammen. Im neuen Ausbildungslehrgang der BITKOM Servicegesellschaft wird das notwendige juristische, technische und organisatorische Grundwissen vermittelt. In der anschließenden sechsmonatigen Coachingphase setzen die Teilnehmer erste Maßnahmen im Unternehmen um und können bei Bedarf auf einen praxiserfahrenen Coach zurückgreifen. Daran anknüpfend bietet die TÜV NORD Akademie - neben weiteren eigenen Ausbildungsgängen zum Thema Datenschutz - den Teilnehmern eine intensive Prüfungsvorbereitung sowie die Zertifizierung zum „Datenschutzbeauftragten (TÜV $\left.{ }^{\circledR}\right)^{\prime \prime}$ an.

Die TÜV Informationstechnik stellt die Referenten für die Intensivprüfungsvorbereitung sowie die Zertifizierungsprüfung. Die TÜViT-Experten sind selbst als Datenschutzbeauftragte tätig und verfügen über eine langjährige Praxiserfahrung. Die aufeinander abgestimmten Schulungsinhalte bieten den Teilnehmern eine umfassende Ausbildung und mit der Zertifizierung einen höheren Mehrwert für den betrieblichen Datenschutzbeauftragten sowie für das Unternehmen, das dessen Fachkompetenz nachweisen kann.

\section{Neues Chipkarten-Betriebssystem von G\&D bietet mehr Sicherheit für elektronische Ausweise}

Giesecke \& Devrient (G\&D) ist der erste Hersteller von Ausweissystemen weltweit, der eine zertifizierte Lösung für die neuen Sicherheitsfunktionen in elektronischen Reisepässen sowie Aufenthaltstiteln und ID-Karten nach dem vom Bundesamt für Sicherheit in der Informationstechnik (BSI) neu definierten Anforderungsprofil anbietet.

Ein entsprechendes Zertifikat hat das BSI dem Münchener Unternehmen jetzt erteilt. Neu an diesem Anforderungsprofil ist die Unterstützung des PACE-Protokolls (Password Authenticated Connection Establishment) jetzt auch bei Pässen. In EU-Ländern wird die Anwendung von PACE in elektronischen Reisepässen und Aufenthaltstiteln ab Dezember 2014 verpflichtend.

Damit erteilt das Bundesamt für Sicherheit in der Informationstechnik (BSI) dem neuen G\&D-Chipkarten-Betriebssystem STARCOS 3.5 das Sicherheitszertifikat nach dem Common Criteria Schutzprofil (BSI-CC-PP-0056-V2-2012) für maschinenlesbare Reisedokumente mit Extended Access Control und PACE.
Der PACE-Sicherheitsmechanismus kommt bereits im neuen deutschen Personalausweis zum Einsatz und G\&D liefert hierfür mit dem Betriebssystem STARCOS 3.5 ebenfalls schon eine zertifizierte Lösung (nach dem ID Card Protection Profile BSI-CC-PP-0061-2009).

Mit dem neuen Chipkarten-Betriebssystem bietet G\&D seinen Kunden bereits jetzt eine sicherheitszertifizierte Lösung, um ihre elektronischen Dokumente auf den neuesten technologischen Stand bringen und wirkungsvoll gegen Missbrauch schützen zu können.

Das neue Sicherheitsprotokoll soll zukünftig nicht nur in hoheitlichen Dokumenten innerhalb der EU-Länder zum Einsatz kommen, sondern weltweit Anwendung finden. So empfiehlt die International Civil Aviation Organisation (ICAO) allen Ländern, ihre biometrischen Reisepässe bis Dezember 2014 auf den PACE-Zugriffsmechanismus umzustellen und damit das Basic Access Control-Protokoll (BAC) abzulösen.

\section{Einstellung des Verkaufs von TC TrustCenter-Produkten und-Services}

Im Juni 2010 hat Symantec durch die Akquisition der PGP Corporation auch TC TrustCenter übernommen, inklusive des in den USA ansässigen Geschäftsbereichs PGP TC, Inc. (zusammenfassend als „TC TrustCenter" bezeichnet). Symantec hat unter Berücksichtigung der Wünsche von Kunden und Partnern überprüft, wie die Anforderungen aller Kunden, einschließlich derer, die aktuell TC TrustCenter-Produkte und -Services verwenden, am besten erfüllt werden können. Der optimale Weg, weiterhin hochwertige Produkte und Services auf dem neuesten Stand bereitzustellen, besteht darin, die vorhandenen Ressourcen auf die aktuellen Angebote der Identitätsund Authentifizierungsservices von Symantec zu konzentrieren.

\section{Einstellung des Verkaufs}

Hiermit (d.h. am 01.08.2012) wird angekündigt, dass am 31. Oktober 2012 alle TC TrustCenter-Produkte und-Services, mit Ausnahme der CI Plus Services, in den Status „End of Sale“ gesetzt werden. Hier finden Sie wichtige Informationen zu unserem Plan, diese Produkte und Services aus dem Verkauf zu nehmen: $h t t p: / / w w w . t r u s t c e n t e r . d e$ /EOS-Notification.htm

Symantec ist es wichtig, allen Kunden Zeit für eine erfolgreiche Umstellung auf alternative Produkte zu ermöglichen. Aus diesem Grund wird der Support für bisherige TC TrustCenter-Kunden über einen angemessenen Zeitraum hinweg aufrechterhalten, um eine möglichst reibungslose Umstellung auf alternative Lösungen zu ermöglichen.

\section{Technischer Support}

Der technische Support wird unverändert beibehalten. TC TrustCenter-Kunden erhalten auch weiterhin denselben Kundensupport, entweder über die TC TrustCenter-Website (https://www.trustcenter. de/infocenter/submitting_inquiries.htm) oder telefonisch.

\section{Signtrust: Mehr Sicherheit mit SSL-Zertifikaten}

Ab dem 01. Juli 2012 werden neu beantragte organisationsgeprüfte SSL-Zertifikate unseres Partners und Zertifikatherausgebers Comodo CA Limited einer zusätzlichen telefonischen Verifizierung des 